\title{
Schlafstörungen bei Multipler Sklerose
}

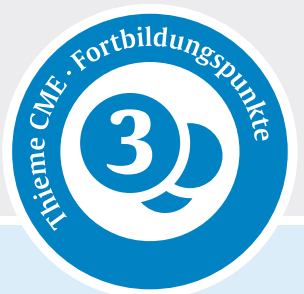

Christian Veauthier

Interdisziplinäres Schlafmedizinisches Zentrum, Charité Universitätsmedizin Berlin, corporate member of Freie Universität Berlin, Humboldt-Universität zu Berlin, and Berlin Institute of Health

\section{ZUSAMMENFASSUNG}

Gegenstand und Ziel: Patienten mit Multipler Sklerose (MS) leiden häufig an einer die Lebensqualität deutlich beeinträchtigenden Schlafstörung. Es soll ein Überblick über den gegenwärtigen Stand der Literatur gegeben werden. Material und Methoden: Übersichtsarbeit. Ergebnisse: Mehrere Studien haben bei der MS im Vergleich zur Allgemeinbevölkerung eine erhöhte Prävalenz für ein Restless-legs-Syndrom (RLS) gezeigt, im Besonderen bei MS-Patienten mit einem erhöhten Grad der Behinderung. Rund ein Viertel der MS-Patienten leidet an ei- ner chronischen Insomnie. Es existieren symptomatische Formen der Narkolepsie und der REM-Schlaf-Verhaltensstörung (RBD), am ehesten durch akute Läsionen hervorgerufen. Ob Läsionen auch schlafbezogene Atmungsstörungen verursachen können, kann noch nicht ausreichend beurteilt werden. Schlussfolgerung und klinische Relevanz: Beim Neuauftreten einer Narkolepsie oder eines RBD sollte zügig eine kraniale Kernspintomografie mit Kontrastmittel erfolgen. Bei der Behandlung schlafmedizinischer Erkrankungen kommen die von den Fachgesellschaften empfohlenen Behandlungsrichtlinien zur Anwendung (die Behandlung unterscheidet sich bei MS-Patienten nicht grundsätzlich von der Behandlung dieser Erkrankungen in der Allgemeinbevölkerung). MS-Fatigue-Patienten sollten niedrigschwellig eine Polygrafie oder Polysomnografie erhalten.

\section{Einleitung}

Die MS ist eine chronische Autoimmunerkrankung, in deren Verlauf es zu einer Demyelinisierung und zu neurodegenerativen Prozessen im zentralen Nervensystem (ZNS) kommt (Gehirn, Optikkussnerv, Rückenmark) [1, 2]. Die MS stellt eine häufige neurologische Erkrankung bei jungen Erwachsenen dar, kann mit einer motorischen Behinderung einhergehen und betrifft häufiger Frauen als Männer [1-3]. Sowohl genetische Ursachen als auch Umweltfaktoren scheinen in der Entstehung der Erkrankung eine Rolle zu spielen [4-9]. Ungefähr drei Viertel aller MS-Patienten leiden unter einer Schlafstörung, welche zur Entstehung einer MS-Fatigue beitragen kann $[10,11]$. Darüber hinaus zeigen MS-Patienten, welche an einer Schlafstörung leiden, eine deutlich reduzierte Lebensqualität im Vergleich zu MS-Patienten ohne Schlafstörung [12].

Der Behandlung einer gleichzeitig vorliegenden Schlafstörung kommt auch deswegen eine besondere Bedeutung zu, da keine nachgewiesen wirksame medikamentöse Therapie zur Behandlung der MS-Fatigue existiert, abgesehen von einer Vitamin-D-Substitution [10, 13-15]. Die Behandlung einer zugrunde liegenden Schlafstörung als Ursache einer Fatigue stellt somit neben der Verhaltenstherapie und physiotherapeutischen Maßnahmen eine der wenigen Möglichkeiten dar, die Fatigue zu bessern $[16,17]$.

\section{Restless-legs-Syndrom}

Die Prävalenz des RLS ist bei MS-Patienten bis zu viermal höher als in der Allgemeinbevölkerung [18-21]. Bis zu 19\% aller MS-Patienten leiden an einem RLS [21]. MS-Patienten, die gleichzeitig an einem RLS leiden, haben verglichen mit MS-Patienten, welche nicht an einem RLS leiden, signifikant häufiger spinale Läsionen [22]. Bei MS-Patienten mit einem höheren Behinderungsgrad und deutlicher Beeinträchtigung der Gehfähigkeit finden sich zudem periodische Beinbewegungen sowohl im NREM (non rapid eye movement)-Schlaf als auch im REM-Schlaf, was für periodische Beinbewegungen untypisch ist und möglicherweise durch infratentorielle oder spinale Läsionen bedingt sein kann [23]. Die Behandlung des RLS sollte bei MS-Patienten genauso erfolgen, wie sie bei Patienten mit einem idiopathischen RLS erfolgt.

\section{Chronische Insomnie}

Die häufigste Schlafstörung bei MS-Patienten stellt die chronische Insomnie dar: Ungefähr ein Viertel aller MS-Patienten leiden unter einer Insomnie [11, 24]. MS-Patientinnen leiden häufiger an einer chronischen Insomnie als männliche MS-Patienten, und die Insomnie geht bei beiden mit einer verringerten Lebensqualität einher [12, 25 , 26]. Allerdings fehlen epidemiologische Studien, welche die Prävalenz der Insomnie bei MS im Vergleich zur Allgemeinbevölkerung untersuchen [27]. In der Literatur finden sich zwar immer wieder Angaben, die Prävalenz der Insomnie sei bei MS erhöht, dies kann jedoch aufgrund 
der schlechten Datenlage nicht als gesichert angesehen werden [28]. Die Selbstmedikation mit freiverkäuflichen Schlaftabletten zur Behandlung der Schlafstörung kann jedoch durch den Überhang dieser Medikamente eine MS-Fatigue vortäuschen oder eine bestehende Fatigue verschlechtern [29, 30]. Die Insomnie geht im Allgemeinen häufig mit Sorgen über die Konsequenzen der Schlafstörungen einher; die Patienten befürchten häufig tagsüber nicht funktionsfähig zu sein, haben häufig die Hoffnung aufgegeben, dass sich die Insomnie bessert und erwarten deswegen eine Besserung von der Einnahme von Schlaftabletten [31, 32]. Allerdings stellen diese dysfunktionalen Annahmen einen der Gründe dafür dar, dass sich die Insomnie nicht bessert und erhalten diese sogar aufrecht [32]. Eine kürzlich veröffentlichte Studie untersuchte vergleichend Insomnie-Patienten aus der Allgemeinbevölkerung und MS-Patienten, welche an einer Insomnie leiden, und in beiden Subgruppen fanden sich keine Unterschiede bezüglich der dysfunktionalen Annahmen [33]. Die Autoren schlussfolgerten, dass sich die Insomnie bei MS nicht wesentlich von der „normalen“ Insomnie in der Allgemeinbevölkerung unterscheidet. Die Behandlung der Insomnie sollte somit analog zu den allgemeinen Leitlinien auch bei MS-Patienten in erster Linie mit kognitiver Verhaltenstherapie erfolgen [34].

\section{Schlafbezogene Atmungsstörungen}

Es existieren keine Studien, die die Prävalenz von schlafbezogenen Atmungsstörungen bei MS im Vergleich zur Allgemeinbevölkerung untersuchen [28]. In Studien mit konsekutiven MS-Patienten wurden Prävalenzen des obstruktiven Schlafapnoesyndroms (OSA) von $0 \%$ bis $58 \%$ gefunden; allerdings wurden dabei verschiedene diagnostische Untersuchungen eingesetzt (Polygrafie oder Polysomnografie), verschiedene Grenzwerte und Klassifikationssysteme verwendet und die Gruppengröße variierte bei diesen Studien erheblich [11, 28, 35-37]. Vor dem Hintergrund, dass Studien im Bereich der Schlaganfallforschung zeigten, dass es nach infratentoriellen Hirninfarkten vermehrt zu zentralen Schlafapnoen kommt, stellt sich die Frage, ob auch infratentorielle Läsionen im Rahmen einer MS zu einer zentralen Schlafapnoe führen können [29, 38-42].

Braley und Mitautoren untersuchten die mögliche Auswirkung von Hirnstammläsionen im Rahmen einer MS auf die Schlafapnoe. In einer retrospektiven Analyse ihrer polysomnografischen Daten von 48 MS Patienten und 48 nicht an einer MS erkrankten Kontrollen der Datenbank des Schlafzenters, fanden die Autoren eine erhöhte Anzahl an zentralen Apnoen bei denjenigen MS-Patienten, die zentrale Hirnstammläsionen aufwiesen, was ein möglicher Hinweis für die Verursachung von zentralen Apnoen durch Hirnstammläsionen darstellt [43]. Allerdings fehlen prospektive, stratifizierte Studien, die diese Zusammenhänge belegen.
Eine Fatigue kann bei MS-Patienten auch auf eine OSA zurückzuführen sein und sich nach einer Überdrucktherapie bessern [16, 17]. Selbst wenn nach den BUB-Richtlinien eine Diagnostik erst bei einer deutlichen Schläfrigkeit vorgesehen ist, sollten MS-Fatigue-Patienten mit einem Punktwert $>34$ in der Modified Fatigue Impact Scale (MFIS) oder einem erhöhten Punktwert $>5$ im Pittsburgh Sleep Quality Index (PSQI) niedrigschwellig eine Polygrafie oder Polysomnografie erhalten [44]. MS-Fatigue-Patienten mit erhöhten Punktwerten im MFIS (>34) oder PSQI (>5) weisen zu 89,8\% eine Schlafstörung auf (Spezifität 58,8\%, positiver prädiktiver Wert $86,3 \%$, negativer prädiktiver Wert $66,7 \%)[44]$.

\section{Narkolepsie und REM-Schlaf-Verhaltens- störung}

In der Literatur wurden 26 Fälle von MS-Patienten, welche an einer Narkolepsie erkrankt sind, beschrieben (bei mehr als 2 Millionen an einer MS erkrankten Patienten weltweit) [45-48]. Sofern eine Bildgebung durchgeführt wurde, finden sich in den meisten Fällen bilaterale Hypothalamusläsionen und bei einigen Patienten im Liquor ein Hypokretindefizit [46]. Bei zwei Patienten mit einem Hypokretindefizit wurde nach einer Cortison-Puls-Therapie Hypokretin im Liquor erneut bestimmt, und es fand sich im Verlauf eine Normalisierung des zuvor verminderten Hypokretinspiegels, was ansonsten bei einer Narkolepsie nicht beschrieben wurde [46]. Diese Remission eines Hypokretindefizits zeigt, dass bei dem Neuauftreten einer Narkolepsie alle MS-Patienten zügig eine kraniale Kernspintomografie mit Kontrastmittel erhalten sollten und dass beim Nachweis ursächlicher akuter Läsionen ohne zeitlichen Verzug eine Cortison-Puls-Therapie erfolgen sollte. Ähnliches wurde auch bei einem RBD beschrieben, mit einer Besserung des RBD durch Behandlung mit ACTH [46].

\section{FAZIT FÜR DIE PRAXIS}

Schlafstörungen sind häufig bei der MS. Ungefähr drei Viertel aller MS-Patienten leiden unter gleichzeitig bestehenden Schlafstörungen [11]. Bei MS-Patienten wurde eine erhöhte Prävalenz für das RLS nachgewiesen im Vergleich zur Allgemeinbevölkerung. Studien, welche die Prävalenz eines OSA und einer Insomnie in MS-Patienten im Vergleich zur Allgemeinbevölkerung untersuchten, fehlen und werden dringend gebraucht. Die Therapie der Schlafstörungen erfolgt bei den einzelnen Schlafstörungen (RLS, Insomnie, OSA, Narkolepsie, RBD) wie in der Allgemeinbevölkerung entsprechend der krankheitsspezifischen Leitlinien resp. Empfehlungen [49-55]. Lediglich die Narkolepsie und das RBD nehmen eine Sonderstellung ein, da bei MS-Patienten mit dem Verdacht auf die Erstmanifestation 
einer dieser beiden Erkrankungen rasch eine Bildgebung und gegebenenfalls eine Schubtherapie (z. B. Cortison-Puls-Therapie) erfolgen sollte, wobei diese Fälle äußerst selten sind. Es bestehen Algorithmen zur Behandlung der MS-Fatigue, bei denen die Behandlung einer zugrunde liegenden Schlafstörung eine besondere Rolle einnimmt [13]. MS-Fatigue-Patienten sollten niedrigschwellig eine Polygrafie oder Polysomnografie erhalten.

Interessenkonflikt

Es bestehen keine Interessenkonflikte.

\section{Korrespondenzadresse}

\section{Dr. med. Christian Veauthier}

Charité Universitätsmedizin - Berlin, Interdisziplinäres Schlafmedizinisches Zentrum, Luisenstraße 13, 10117 Berlin Tel. 030/450513232, Fax 030/450513959

christian.veauthier@charite.de

\section{Literatur}

[1] Goodin DS. The causal cascade to multiple sclerosis: a model for MS pathogenesis. PLoS ONE 2009; 4(2): e4565.

[2] Borisow N, Döring A, Pfueller CF, Paul F, Dörr J, Hellwig K. Expert recommendations to personalization of medical approaches in treatment of multiple sclerosis: an overview of family planning and pregnancy. EPMA Journal 2012; 3(1): 9.

[3] Sinnecker T, Mittelstaedt P, Dörr J, Pfueller CF, Harms L, Niendorf T, et al. Multiple sclerosis lesions and irreversible brain tissue damage: a comparative ultrahigh-field strength magnetic resonance imaging study. Arch Neurol 2012; 69(6): 739-45.

[4] Behrens J, Pfüller C, Mansow-Model S, Otte K, Paul F, Brandt AU. Using perceptive computing in multiple sclerosis - the Short Maximum Speed Walk test. J Neuroeng Rehabil 2014; 11: 89.

[5] Dörr ], Döring A, Paul F. Can we prevent or treat multiple sclerosis by individualised vitamin D supply? EPMA J 2013; $4(1): 4$.

[6] Endriz J, Ho PP, Steinman L. Time correlation between mononucleosis and initial symptoms of MS. Neurol Neuroimmunol Neuroinflamm 2017; 4(3): e308.

[7] Pfuhl C, Oechtering J, Rasche L, Gieß RM, Behrens JR, Wakonig K, et al. Association of serum Epstein-Barr nuclear antigen-1 antibodies and intrathecal immunoglobulin synthesis in early multiple sclerosis. J Neuroimmunol 2015; 285: 156-60.

[8] Srinivasan S, Di Dario M, Russo A, Menon R, Brini E, Romeo $\mathrm{M}$, et al. Dysregulation of MS risk genes and pathways at dis- tinct stages of disease. Neurol Neuroimmunol Neuroinflamm 2017; 4(3): e337.

[9] Zivadinov R, Cerza N, Hagemeier ], Carl E, Badgett D, Ramasamy DP, et al. Humoral response to EBV is associated with cortical atrophy and lesion burden in patients with MS. Neurol Neuroimmunol Neuroinflamm 2016; 3(1): e190.

[10] Veauthier C. Fatigue bei Multipler Sklerose: Mögliche Ursachen und Zusammenhänge, sinnvolle Diagnostik und Therapie. Nervenheilkunde 2011; 30(07): 470-5.

[11] Veauthier C, Radbruch H, Gaede G, Pfueller C, Dorr J, Bellmann-Strobl J, et al. Fatigue in multiple sclerosis is closely related to sleep disorders: a polysomnographic cross-sectional study. Multiple Sclerosis Journal 2011; 17(5): 613-22.

[12] Veauthier C, Gaede G, Radbruch H, Wernecke K-D, Paul F. Sleep Disorders Reduce Health-Related Quality of Life in Multiple Sclerosis (Nottingham Health Profile Data in Patients with Multiple Sclerosis). International Journal of Molecular Sciences 2015; 16(7): 16514-28.

[13] Veauthier C, Hasselmann H, Gold SM, Paul F. The Berlin Treatment Algorithm: recommendations for tailored innovative therapeutic strategies for multiple sclerosis-related fatigue. EPMA J 2016; 7: 25.

[14] Achiron A, Givon U, Magalashvili D, Dolev M, Liraz Zaltzman $\mathrm{S}$, Kalron A, et al. Effect of Alfacalcidol on multiple sclerosis-related fatigue: A randomized, double-blind placebo-controlled study. Multiple Sclerosis Journal 2015; 21(6): 767-75.

[15] Branas P. Treatments for fatigue in multiple sclerosis: a rapid and systematic review. [cited 2015 Dec 5]; Available from: http://www.hta.ac.uk/1167.

[16] Veauthier C, Gaede G, Radbruch H, Gottschalk S, Wernecke K-D, Paul F. Treatment of sleep disorders may improve fatigue in multiple sclerosis. Clinical Neurology and Neurosurgery 2013; 115(9): 1826-30.

[17] Côté I, Trojan DA, Kaminska M, Cardoso M, Benedetti A, Weiss $D$, et al. Impact of sleep disorder treatment on fatigue in multiple sclerosis. Mult Scler 2013; 19(4): 480-9.

[18] Manconi M, Rocca MA, Ferini-Strambi L, Tortorella P, Agosta $\mathrm{F}$, Comi $\mathrm{G}$, et al. Restless legs syndrome is a common finding in multiple sclerosis and correlates with cervical cord damage. Mult Scler 2008; 14(1): 86-93.

[19] Li Y, Munger KL, Batool-Anwar S, De Vito K, Ascherio A, Gao X. Association of multiple sclerosis with restless legs syndrome and other sleep disorders in women. Neurology 2012; 78(19): 1500-6.

[20] Miri S, Rohani M, Sahraian MA, Zamani B, Shahidi GA, Sabet $A$, et al. Restless legs syndrome in Iranian patients with multiple sclerosis. Neurol Sci 2013; 34(7): 1105-8.

[21] Italian REMS Study Group, Manconi M, Ferini-Strambi L, Filippi M, Bonanni E, ludice A, et al. Multicenter case-control study on restless legs syndrome in multiple sclerosis: the REMS study. Sleep 2008; 31(7): 944-52.

[22] Minár M, Petrleničová D, Valkovič P. Higher prevalence of restless legs syndrome/Willis-Ekbom disease in multiple sclerosis patients is related to spinal cord lesions. Mult Scler Relat Disord 2017; 12: 54-8.

[23] Veauthier C, Gaede G, Radbruch H, Sieb J-P, Wernecke K-D, Paul F. Periodic limb movements during REM sleep in multiple sclerosis: a previously undescribed entity. Neuropsychiatric Disease and Treatment 2015; 23: 23.

[24] Viana P, Rodrigues E, Fernandes C, Matas A, Barreto R, Mendonça M, et al. InMS: Chronic insomnia disorder in multiple sclerosis - a Portuguese multicentre study on prevalence, subtypes, associated factors and impact on quality of life. Mult Scler Relat Disord 2015; 4(5): 477-83. 
[25] Ferreira LN, Ferreira PL, Pereira LN, Oppe M. EQ-5D Portuguese population norms. Qual Life Res 2014; 23(2): 425-30.

[26] Dolan P. Modeling valuations for EuroQol health states. Med Care 1997; 35(11): 1095-108.

[27] Vitkova M, Rosenberger ], Gdovinova Z, Szilasiova J, Mikula P, Groothoff JW, et al. Poor sleep quality in patients with multiple sclerosis: gender differences. Brain and Behavior 2016; 6(11): e00553.

[28] Veauthier C, Paul F. Sleep disorders in multiple sclerosis and their relationship to fatigue. Sleep Medicine 2014; 15(1): 5-14.

[29] Braley TJ, Segal BM, Chervin RD. Hypnotic use and fatigue in multiple sclerosis. Sleep Medicine 2015; 16(1): 131-7.

[30] Veauthier C. Hypnotic use and multiple sclerosis related fatigue: a forgotten confounder. Sleep Medicine 2015; 16(3): 319.

[31] Morin CM, Stone J, Trinkle D, Mercer J, Remsberg S. Dysfunctional beliefs and attitudes about sleep among older adults with and without insomnia complaints. Psychol Aging 1993; 8(3): 463-7.

[32] Morin AK, Jarvis Cl, Lynch AM. Therapeutic options for sleep-maintenance and sleep-onset insomnia. Pharmacotherapy $2007 ; 27(1): 89-110$.

[33] Schellaert V, Labauge P, Lebrun C, Maudarbocus KH, Bernard J, Blache J-B, et al. Psychological processes associated with insomnia in patients with multiple sclerosis. Sleep 2018; 41(3).

[34] de Bruin E], Bögels SM, Oort F], Meijer AM. Efficacy of Cognitive Behavioral Therapy for Insomnia in Adolescents: A Randomized Controlled Trial with Internet Therapy, Group Therapy and A Waiting List Condition. Sleep 2015; 38(12): 1913-26.

[35] Kaminska M, Kimoff R, Benedetti A, Robinson A, Bar-Or A, Lapierre $Y$, et al. Obstructive sleep apnea is associated with fatigue in multiple sclerosis. Multiple Sclerosis Journal 2012; 18(8): 1159-69.

[36] Kallweit U, Baumann CR, Harzheim M, Hidalgo H, Pöhlau D, Bassetti CL, et al. Fatigue and Sleep-Disordered Breathing in Multiple Sclerosis: A Clinically Relevant Association? Multiple Sclerosis International 2013; 2013: 1-7.

[37] Chen J-H, Liu X-Q, Sun H-Y, Huang Y. Sleep Disorders in Multiple Sclerosis in China: Clinical, Polysomnography Study, and Review of the Literature. Journal of Clinical Neurophysiology 2014; 31(4): 375-81.

[38] Blissitt PA. Sleep-Disordered Breathing After Stroke: Nursing Implications. Stroke 2017; 48(3): e81-4.

[39] Bogousslavsky J, Khurana R, Deruaz JP, Hornung JP, Regli $F$, Janzer $R$, et al. Respiratory failure and unilateral caudal brainstem infarction. Ann Neurol 1990; 28(5): 668-73.

[40] Devereaux MW, Keane JR, Davis RL. Automatic respiratory failure associated with infarction of the medulla. Report of two cases with pathologic study of one. Arch Neurol 1973; 29(1): 46-52.

[41] Levin BE, Margolis G. Acute failure of automatic respirations secondary to a unilateral brainstem infarct. Ann Neurol 1977; 1(6): 583-6.

[42] Lyons OD, Ryan CM. Sleep Apnea and Stroke. Can J Cardiol 2015; 31(7): 918-27.

[43] Braley T], Segal BM, Chervin RD. Sleep-disordered breathing in multiple sclerosis. Neurology 2012; 79(9): 929-36.
[44] Veauthier C, Paul F. Fatigue in multiple sclerosis: which patient should be referred to a sleep specialist? Multiple Sclerosis Journal 2012; 18(2): 248-9.

[45] Kallweit U, Bassetti CLA, Oberholzer M, Fronczek R, Béguin $\mathrm{M}$, Strub M, et al. Coexisting narcolepsy (with and without cataplexy) and multiple sclerosis: Six new cases and a literature review. J Neurol 2018; 265(9): 2071-8.

[46] Veauthier C. Sleep Disorders in Multiple Sclerosis. Review. Current Neurology and Neuroscience Reports [Internet]. 2015; 15(5). Available from: http://link.springer. com/10.1007/s11910-015-0546-0.

[47] Browne P, Chandraratna D, Angood C, Tremlett H, Baker C, Taylor BV, et al. Atlas of Multiple Sclerosis 2013: A growing global problem with widespread inequity. Neurology 2014; 83(11): 1022-4.

[48] Evans C, Beland S-G, Kulaga S, Wolfson C, Kingwell E, Marriott J, et al. Incidence and Prevalence of Multiple Sclerosis in the Americas: A Systematic Review. Neuroepidemiology 2013; 40(3): 195-210.

[49] Riemann D, Baglioni C, Bassetti C, Bjorvatn B, Dolenc Groselj L, Ellis JG, et al. European guideline for the diagnosis and treatment of insomnia. J Sleep Res 2017 Sep 5; E-pub.

[50] Aurora RN, Kristo DA, Bista SR, Rowley JA, Zak RS, Casey KR, et al. Update to the AASM Clinical Practice Guideline: “The Treatment of Restless Legs Syndrome and Periodic Limb Movement Disorder in Adults-An Update for 2012: Practice Parameters with an Evidence-Based Systematic Review and Meta-Analyses." SLEEP [Internet]. 2012 Aug 1 [cited 2015 Nov 26]; Available from: http://www.journalsleep.org/ViewAbstract.aspx?pid $=28591$

[51] Sateia M], Buysse D], Krystal AD, Neubauer DN, Heald JL. Clinical Practice Guideline for the Pharmacologic Treatment of Chronic Insomnia in Adults: An American Academy of Sleep Medicine Clinical Practice Guideline. J Clin Sleep Med 2017; 13(2): 307-49.

[52] Högl B, Stefani A. REM sleep behavior disorder (RBD): Update on diagnosis and treatment. Somnologie 2017; 21(Suppl 1): $1-8$.

[53] Billiard M, Bassetti C, Dauvilliers Y, Dolenc-Groselj L, Lammers G], Mayer G, et al. EFNS guidelines on management of narcolepsy. Eur J Neurol 2006; 13(10): 1035-48.

[54] Richtlinie des Gemeinsamen Bundesausschusses zu Untersuchungs- und Behandlungsmethoden der vertragsärztlichen Versorgung. Berlin: Bundesanzeiger 2016.

[55] Garcia-Borreguero D, Silber MH, Winkelman JW, Högl B, Bainbridge J, Buchfuhrer M, et al. Guidelines for the first-line treatment of restless legs syndrome/Willis-Ekbom disease, prevention and treatment of dopaminergic augmentation: a combined task force of the IRLSSG, EURLSSG, and the RLS-foundation. Sleep Med 2016; 21: 1-11.

\section{Bibliografie}

DOI https://doi.org/10.1055/a-0817-2588

Nervenheilkunde 2019; 38: 97-100

(c) Georg Thieme Verlag KG Stuttgart · New York

ISSN 0722-1541 


\section{Punkte sammeln auf CME.thieme.de}

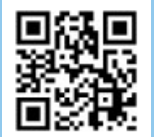

Diese Fortbildungseinheit ist in der Regel 12 Monate online für die Teilnahme verfügbar. Den genauen Einsendeschluss finden Sie unter https://eref.thieme.de/CXCHLWM.

Sollten Sie Fragen zur Online-Teilnahme haben, finden Sie unter https://cme.thieme.de/hilfe eine ausführliche Anleitung. Wir wünschen viel Erfolg beim Beantworten der Fragen!

Unter https://eref.thieme.de/CXCHLWM oder über den QR-Code kommen Sie direkt zur Startseite des Wissenstests.

VNR 2760512019156645325

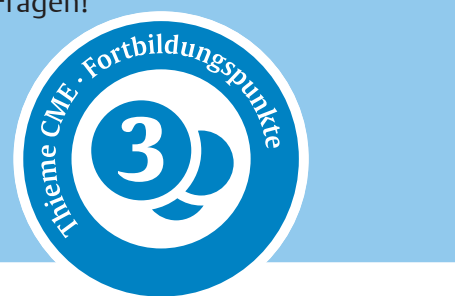

\section{Frage 1}

Lebensqualität/Fatigue und Schlafstörungen bei MS. Welche Antwort ist richtig?

A MS-Patienten, welche an einer Schlafstörung leiden, zeigen eine deutlich reduzierte Lebensqualität im Vergleich zu MS-Patienten ohne Schlafstörung.

B MS-Patienten, welche an einer Schlafstörung leiden, zeigen eine unveränderte Lebensqualität im Vergleich zu MS-Patienten ohne Schlafstörung.

C MS-Patienten, welche an einer Schlafstörung leiden, zeigen eine verbesserte Lebensqualität im Vergleich zu MS-Patienten ohne Schlafstörung.

D Es gibt bislang keine Studien über den Zusammenhang zwischen dem Vorliegen einer Schlafstörung und der Lebensqualität bei MS-Patienten.

E Die MS-Fatigue wird durch die Behandlung einer zugrunde liegenden Schlafstörung nicht gebessert.

\section{Frage 2}

RLS und MS. Welche Antwort ist richtig?

A Die Prävalenz des RLS ist bei MS-Patienten bis zu 10-mal höher als in der Allgemeinbevölkerung.

B Die Prävalenz des RLS ist bei MS-Patienten bis zu 4-mal höher als in der Allgemeinbevölkerung.

C Die Prävalenz des RLS ist bei MS-Patienten doppelt so hoch wie in der Allgemeinbevölkerung.

D Die Prävalenz des RLS ist bei MS-Patienten genauso hoch wie in der Allgemeinbevölkerung.

E Die Prävalenz des RLS ist bei MS-Patienten halb so hoch wie in der Allgemeinbevölkerung.

\section{Frage 3}

Häufigkeit des RLS bei MS-Patienten. Welche Antwort ist richtig?

A Bis zu $50 \%$ aller MS-Patienten leiden an einem RLS.

B Bis zu 33 \% aller MS-Patienten leiden an einem RLS.

C Bis zu $19 \%$ aller MS-Patienten leiden an einem RLS.

D Bis zu $9 \%$ aller MS-Patienten leiden an einem RLS.

E Bis zu $4 \%$ aller MS-Patienten leiden an einem RLS.

\section{Frage 4}

RLS und MS-Läsionen. Welche Antwort ist richtig?

A MS-Patienten, die gleichzeitig an einem RLS leiden, haben verglichen mit MS-Patienten, welche nicht an einem RLS leiden, signifikant seltener periventriculäre Läsionen.

B MS-Patienten, die gleichzeitig an einem RLS leiden, haben verglichen mit MS-Patienten, welche nicht an einem RLS leiden, signifikant häufiger Läsionen im Balken.

C MS-Patienten, die gleichzeitig an einem RLS leiden, haben verglichen mit MS-Patienten, welche nicht an einem RLS leiden, signifikant seltener zerebelläre Läsionen.

D MS-Patienten, die gleichzeitig an einem RLS leiden, haben verglichen mit MS-Patienten, welche nicht an einem RLS leiden, signifikant häufiger spinale Läsionen.

E MS-Patienten, die gleichzeitig an einem RLS leiden, haben verglichen mit MS-Patienten, welche nicht an einem RLS leiden, signifikant seltener T1-Hypointensitäten („black holes“).

\section{Frage 5}

Behandlung des RLS bei MS. Welche Antwort ist richtig?

A Die Behandlung des RLS bei MS erfolgt nach einem speziellen „RLS bei MS“-Therapie-Schema/Algorithmus.

B Die Behandlung des RLS sollte bei MS-Patienten genauso erfolgen, wie sie bei Patienten mit einem idiopathischen RLS erfolgt.

C Ein RLS muss bei MS-Patienten nicht behandelt werden, sondern es sollte nur die MS behandelt werden.

D Zur Behandlung des RLS bei MS sollten keine Dopaminagonisten verordnet werden.

E Dopaminagonisten sind zur Behandlung des RLS bei MS nicht zugelassen.

\footnotetext{
- Weitere Fragen auf der folgenden Seite ...
} 
Punkte sammeln auf CMF.thieme.de

Fortsetzung ...

\section{Frage 6}

Insomnie und MS. Welche Antwort ist richtig?

A Es gibt epidemiologische Studien, welche die Prävalenz einer chronischen Insomnie bei MS im Vergleich zur Allgemeinbevölkerung untersuchten.

B Ungefähr die Hälfte aller MS-Patienten leiden unter einer Insomnie.

C Ungefähr 10 \% aller MS-Patienten leiden unter einer Insomnie.

D MS-Patienten leiden selten unter einer chronischen Insomnie.

E Die häufigste Schlafstörung bei MS-Patienten stellt die chronische Insomnie dar.

\section{Frage 7}

Geschlechtsunterschiede bei der Insomnie bei MS. Welche Antwort ist richtig?

A MS-Patienten leiden häufiger an einer chronischen Insomnie als MS-Patientinnen.

B Es gibt keine Geschlechtsunterschiede bzgl. einer chronischen Insomnie bei MS.

C MS-Patientinnen leiden häufiger an einer chronischen Insomnie als MS-Patienten.

D Die Insomnie geht bei MS-Patienten mit einer verbesserten Lebensqualität einher.

E Dysfunktionale Annahmen (Sorgen über die Konsequenzen der Schlafstörungen und dass sich die chronische Insomnie nicht bessern wird) spielen bei MS-Patienten keine Rolle.

\section{Frage 8}

Behandlung der Insomnie bei MS. Welche Antwort ist richtig?

A MS-Patienten, welche an einer chronischen Insomnie erkrankt sind, sollten nicht mit kognitiver Verhaltenstherapie behandelt werden.

B Die Selbstmedikation mit freiverkäuflichen Schlaftabletten zur Behandlung der Schlafstörung kann eine MS-Fatigue vortäuschen oder eine bestehende Fatigue verschlechtern.

C Die Behandlung der Insomnie sollte bei MS-Patienten nicht anhand der allgemeinen Leitlinien zur Behandlung der chronischen Insomnie erfolgen.

D Bei MS-Patienten spielen dysfunktionale Annahmen bei der Entwicklung und Aufrechterhaltung der Insomnie keine Rolle.

E Die Insomnie bei MS braucht nicht behandelt zu werden.

\section{Frage 9}

Schlafapnoe und MS. Welche Antwort ist richtig?

A Zwischen einer Schlafapnoe und der MS-Fatigue besteht kein Zusammenhang, da es sich um verschiedene Erkrankungen handelt.

B Es existieren methodisch gute, repräsentative epidemiologische Studien, welche belegen, dass eine Schlafapnoe bei MS-Patienten häufiger ist als in der Allgemeinbevölkerung.

C Bei MS-Patienten, welche vermehrt infratentorielle Läsionen aufweisen und an einer Schlafapnoe erkrankt sind, kommt es seltener zu zentralen Apnoen.

D Es existieren epidemiologische Studien, die die Prävalenz von schlafbezogenen Atmungsstörungen bei MS im Vergleich zur Allgemeinbevölkerung untersuchen.

E Eine Fatigue kann bei MS-Patienten auch auf eine Schlafapnoe zurückzuführen sein und sich nach einer Überdrucktherapie (CPAP) bessern.

\section{Frage 10}

Narkolepsie, RBD und MS. Welche Antwort ist richtig?

A Es gibt Fallberichte, welche zeigten, dass sich bei Patienten mit einer Narkolepsie und einer MS das Hypokretindefizit nach einer Cortison-Puls-Therapie gebessert hat.

B Bei MS Patienten ist eine kraniale Kernspintomografie nach der Erstmanifestation einer Narkolepsie nicht notwendig, da die Narkolepsie in diesem Fall autoimmun bedingt ist.

C Beim Nachweis einer für eine Narkolepsie ursächlichen Läsion sollte keine Cortison-Puls-Therapie durchgeführt werden.

D Die Prävalenz der Narkolepsie ist höher als die Prävalenz der MS.

E Es gibt keine Fälle in der Literatur, die zeigen, dass MS-Patienten auch an einer Narkolepsie erkranken können. 